\title{
On the Equality of Rank of a Fourth-Idempotent Matrix
}

\author{
Lin Deng ${ }^{1 *}$, Feng Zhang ${ }^{2}$, Jinli $\mathrm{Xu}^{3}$ \\ Department of mathematics, Northeast Forestry University, Harbin, China, 150040
}

*Corresponding Author: Lin Deng, Depar tment of mathematics, Northeast Forestry University, Harbin, China, 150040

\begin{abstract}
The equality of rank a fourth-idempotent matrix is established by means of elementary transformation and properties of idempotent matrix.
\end{abstract}

Keywords: fourth-idempotent matrix, rank, equality.

\section{INTRODUCTION}

Proposition $1 A^{4}=A \Leftrightarrow \operatorname{rank}(A)+\operatorname{rank}\left(E-A^{3}\right)=n$

Proof: Since the elementary transformation of a matrix does not change the rank of the matrix, the following equality can be obtained.

$$
\operatorname{rank}\left[\begin{array}{cc}
A & \\
& E-A^{3}
\end{array}\right]=\operatorname{rank}\left[\begin{array}{cc}
A & \\
A^{3} & E-A^{3}
\end{array}\right]=\operatorname{rank}\left[\begin{array}{cc}
A & A \\
A^{3} & E
\end{array}\right]=\operatorname{rank}\left[\begin{array}{cc}
A-A^{4} & 0 \\
A^{3} & E
\end{array}\right]=\operatorname{rank}\left[\begin{array}{cc}
A-A^{4} & 0 \\
0 & E
\end{array}\right]
$$

Therefore $A^{4}=A \Leftrightarrow \operatorname{rank}(A)+\operatorname{rank}\left(E-A^{3}\right)=n$

Proposition $2 A^{4}=A \Rightarrow \operatorname{rank}\left(A^{a}\right)+\operatorname{rank}\left(E-A^{3}\right)^{b}=n$

Proof: On the one hand, by $A^{4}=A$, we have $A\left(E-A^{3}\right)=0$, So for every positive integer, we have $A^{a}\left(E-A^{3}\right)=0$. With the help of the property of matrix multiplication operation, we can get $\operatorname{rank}\left(A^{a}\right)+\operatorname{rank}\left(E-A^{3}\right) \leq n$.

On the other hand, The minimum polynomial of matrix $A$ obtained from $A^{4}=A$ is the factor of polynomial $\lambda^{4}-\lambda$. Therefore, the minimum polynomial of $\boldsymbol{A}$ has no multiple roots, so $A$ can be diagonalized.

For every positive integer $a, b$, there exists an invertible matrix $P$ such that the following equation holds. $P\left[A^{a}+\left(E-A^{3}\right)^{b}\right] P^{-1}=P A^{a} P^{-1}+P\left(E-A^{3}\right)^{b} P^{-1}=\left(P A P^{-1}\right)^{a}+\left[E-\left(P A P^{-1}\right)^{3}\right]^{b}$

It is not hard to get $\operatorname{rank}\left[A^{a}+\left(E-A^{3}\right)^{b}\right]=n$. Hence it follows that

$n=\operatorname{rank}\left[A^{a}+\left(E-A^{3}\right)^{b}\right] \leq \operatorname{rank}\left(A^{a}\right)+\operatorname{rank}\left(E-A^{3}\right)^{b} \leq n$

Therefore $\operatorname{rank}\left(A^{a}\right)+\operatorname{rank}\left(E-A^{3}\right)^{b}=n$

Conversely, it does not necessarily hold true. Here's an example. $A=\left[\begin{array}{lll}1 & 1 & 0 \\ & 1 & 1 \\ & & 1\end{array}\right]$, when $a=4, b=4$, $\operatorname{rank}\left(A^{a}\right)+\operatorname{rank}\left(E-A^{3}\right)^{b}=3$, but $A^{4} \neq A$

Proposition $3 A^{4}=A \Rightarrow \operatorname{rank}(A)+\operatorname{rank}\left(E-A^{3}+A^{2}\right)=n+\operatorname{rank}\left(A^{3}\right)$

Proof The following equation can be obtained from elementary transformation. 


$$
\begin{aligned}
& \operatorname{rank}\left[\begin{array}{ll}
A & \\
& E-A^{3}+A^{2}
\end{array}\right]=\operatorname{rank}\left[\begin{array}{ll}
A & \\
A & E-A^{3}+A^{2}
\end{array}\right]=\operatorname{rank}\left[\begin{array}{cc}
A & A^{3}-A^{2} \\
A & E
\end{array}\right] \\
& =\operatorname{rank}\left[\begin{array}{cc}
A-A^{4}+A^{3} & A^{3}-A^{2} \\
0 & E
\end{array}\right]=\operatorname{rank}\left[\begin{array}{cc}
A-A^{4}+A^{3} & 0 \\
0 & E
\end{array}\right],
\end{aligned}
$$

Substituting $A^{4}=A, \operatorname{rank}\left[\begin{array}{cc}A-A^{4}+A^{3} & 0 \\ 0 & E\end{array}\right]=\operatorname{rank}\left[\begin{array}{cc}A^{3} & 0 \\ 0 & E\end{array}\right]$, Therefore $\operatorname{rank}\left[\begin{array}{cc}A & \\ & E-A^{3}+A^{2}\end{array}\right]=\operatorname{rank}\left[\begin{array}{rr}A^{3} & 0 \\ 0 & E\end{array}\right]$. That is to say $\operatorname{rank}(A)+\operatorname{rank}\left(E-A^{3}+A^{2}\right)=n+\operatorname{rank}\left(A^{3}\right)$

Conversely, it does not necessarily hold true. For example, $A=3 E$, $\operatorname{rank}(A)+\operatorname{rank}\left(E-A^{3}+A^{2}\right)=2 n=n+\operatorname{rank}\left(A^{3}\right)$, but $A^{4}=81 E \neq 3 E=A$.

Proposition $4 A^{4}=A \Rightarrow \operatorname{rank}\left(E-A^{3}+A^{2}\right)=\operatorname{rank}\left(A^{3}\right)+\operatorname{rank}\left(E-A^{3}\right)$

$A^{4}=A, \operatorname{rank}(A)+\operatorname{rank}\left(E-A^{3}+A^{2}\right)=n+\operatorname{rank}\left(A^{3}\right), \operatorname{rank}(A)+\operatorname{rank}\left(E-A^{3}\right)=n$,

So we can get $\operatorname{rank}\left(E-A^{3}+A^{2}\right)=\operatorname{rank}\left(A^{3}\right)+\operatorname{rank}\left(E-A^{3}\right)$.

But the same, based on $\operatorname{rank}\left(E-A^{3}+A^{2}\right)=\operatorname{rank}\left(A^{3}\right)+\operatorname{rank}\left(E-A^{3}\right)$, we can not get $A^{4}=A$. For example,

$$
A=\left[\begin{array}{lll}
1 & 1 & 0 \\
0 & 0 & 1 \\
0 & 0 & 0
\end{array}\right], A^{2}=A^{3}=\left[\begin{array}{lll}
1 & 1 & 1 \\
0 & 0 & 0 \\
0 & 0 & 0
\end{array}\right]
$$

$\operatorname{rank}\left(E-A^{3}+A^{2}\right)=3=\operatorname{rank}\left(A^{3}\right)+\operatorname{rank}\left(E-A^{3}\right)$, but $A^{4}=\left[\begin{array}{lll}1 & 1 & 1 \\ 0 & 0 & 0 \\ 0 & 0 & 0\end{array}\right] \neq\left[\begin{array}{lll}1 & 1 & 0 \\ 0 & 0 & 1 \\ 0 & 0 & 0\end{array}\right]=A$

According to the definition of the fourth-idempotent matrix and its operation, the following properties of the fourth-idempotent matrix can be given.

\section{Proposition 4}

(1)If the fourth-idempotent matrices $A, B$ are commutative, then $A B$ is also a fourth-idempotent matrix.

(2) If $A$ is a fourth-idempotent matrix, then $A^{3}$ is an idempotent matrix.

(3) If $A$ is a fourth-idempotent matrix, $E-A^{3}$ is an idempotent matrix.

(4) If $A$ is a fourth-idempotent matrix, then for any positive integer, there are $A^{n}=\left\{\begin{array}{c}A, 3 \mid n-1 \\ A^{2}, 3 \mid n-2 \\ A^{3}, 3 \mid n\end{array}\right.$

Proposition 5 If $A, B$ are all fourth-idempotent matrices, the following equality is satisfied

1) $\operatorname{rank}\left(A^{3}+B^{3}\right)=\operatorname{rank}\left[\begin{array}{cc}A^{3} & B^{3} \\ B^{3} & 0\end{array}\right]-\operatorname{rank} B^{3}=\operatorname{rank}\left[\begin{array}{cc}B^{3} & A^{3} \\ A^{3} & 0\end{array}\right]-\operatorname{rank} A^{3}$

(2) $\operatorname{rank}\left(A^{3}+B^{3}\right)=\operatorname{rank}\left[A^{3}-A^{3} B^{3}, B^{3}\right]=\operatorname{rank}\left[B^{3}-B^{3} A^{3}, A^{3}\right]$

(3) $\operatorname{rank}\left(A^{3}+B^{3}\right)=\operatorname{rank}\left(A^{3}-A^{3} B^{3}-B^{3} A^{3}+B^{3} A^{3} B^{3}\right)+\operatorname{rank} B^{3}$

(4) $\operatorname{rank}\left(A^{3}+B^{3}\right)=\operatorname{rank}\left(A^{3}-A^{3} B^{3}-B^{3} A^{3}+A^{3} B^{3} A^{3}\right)+\operatorname{rank} A^{3}$ 
(5) $\operatorname{rank}\left(A^{3}+B^{3}\right)=\operatorname{rank}\left[\begin{array}{ccc}A^{3} & B^{3} & 0 \\ B^{3} & 0 & A^{3}\end{array}\right]=\operatorname{rank}\left[\begin{array}{cc}A^{3} & B^{3}\end{array}\right]$

(6)If $a_{1}, a_{2}$ are two non-zero real numbers and $a_{1}+a_{2} \neq 0$, then $\operatorname{rank}\left(a_{1} A^{3}+a_{2} B^{3}\right)=\operatorname{rank}\left(A^{3}+B^{3}\right)$.

Theorem $1 A \in P^{n \times n}, f(x) \in P[x]$ is a polynomial with any number greater than 1 . Let

$d(x)=\left(f(x), x-x^{4}\right)$ and $m(x)=\left[f(x), x-x^{4}\right]$, then

$\operatorname{rankf}(A)+\operatorname{rank}\left(A-A^{4}\right)=\operatorname{rankd}(A)+\operatorname{rankm}(A)$.

With the help of theorem 1 we can get if $\boldsymbol{A}$ is a fourth-idempotent matrix, then

$\operatorname{rankf}(A)=\operatorname{rankd}(A)+\operatorname{rankm}(A)$

This theorem shows that there are also many rank eigenvalues of fourth-idempotent matrices.

\section{Theorem 2}

$A \in P^{n \times n}, t \geq 1 \in N^{+}, \operatorname{rank}(A)+\operatorname{rank}\left(A^{t}-A^{t+3}\right)=\operatorname{rank}\left(A^{t}\right)+\operatorname{rank}\left(A-A^{4}\right)$.

Proof: When $\mathrm{t}=1$, the equation clearly holds.

Let $t>1, f(x)=x^{t}, \quad g(x)=x-x^{4}$, By simple calculation we get $(f(x), g(x))=x$ $[f(x), g(x)]=x^{t}-x^{t+3}$. By the above we can get the following equation.

$\operatorname{rank}(A)+\operatorname{rank}\left(A^{t}-A^{t+3}\right)=\operatorname{rank}\left(A^{t}\right)+\operatorname{rank}\left(A-A^{4}\right)$

\section{REFERENCES}

[1] Baksalary O M, Bernstein D S, Trenkler G. On the equality between rank and trace of an idempotent matrix[J]. Applied Mathematics \& Computation, 2010, 217(8):4076-4080.

[2] Wright S E. A note on the equality of rank and trace for an idempotent matrix[J]. Applied Mathematics \& Computation, 2011, 217(16):7048-7049.

[3] Tian Y, Styan G P H. Rank equalities for idempotent matrices with applications[M]. 2006.

[4] Baksalary J K, Baksalary O M, Szulc T. Properties of Schur complements in partitioned idempotent matrices[J]. Linear Algebra \& Its Applications, 2004, 379(1):303-318.

Citation: Lin Deng, et.al., (2019). On the Equality of Rank of a Fourth-Idempotent Matrix. International Journal of Scientific and Innovative Mathematical Research (IJSIMR), 7(5), pp.1-3. http://dx.doi.org/ $10.20431 / 2347-3142.0705001$

Copyright: (C) 2019 Authors, this is an open-access article distributed under the terms of the Creative Commons Attribution License, which permits unrestricted use, distribution, and reproduction in any medium, provided the original author and source are credited. 